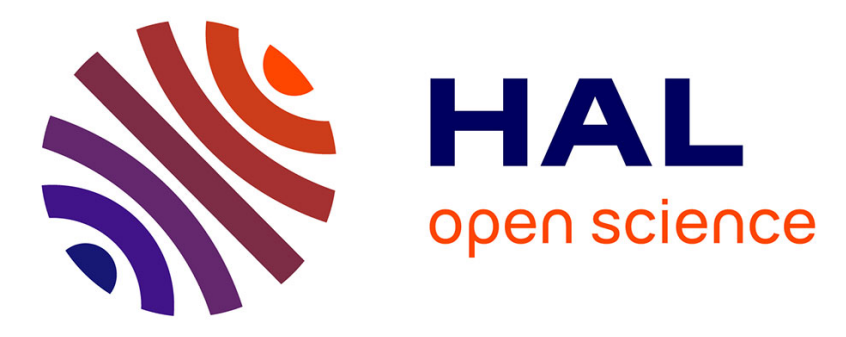

\title{
Towards Haptic Images: a Survey on Touchscreen-Based Surface Haptics
}

Antoine Costes, Fabien Danieau, Ferran Argelaguet Sanz, Philippe Guillotel, Anatole Lécuyer

\section{- To cite this version:}

Antoine Costes, Fabien Danieau, Ferran Argelaguet Sanz, Philippe Guillotel, Anatole Lécuyer. Towards Haptic Images: a Survey on Touchscreen-Based Surface Haptics. IEEE Transactions on Haptics (ToH), 2020, 13 (3), pp.530-541. 10.1109/TOH.2020.2984754 . hal-03007468

\section{HAL Id: hal-03007468 \\ https://hal.inria.fr/hal-03007468}

Submitted on 16 Nov 2020

HAL is a multi-disciplinary open access archive for the deposit and dissemination of scientific research documents, whether they are published or not. The documents may come from teaching and research institutions in France or abroad, or from public or private research centers.
L'archive ouverte pluridisciplinaire HAL, est destinée au dépôt et à la diffusion de documents scientifiques de niveau recherche, publiés ou non, émanant des établissements d'enseignement et de recherche français ou étrangers, des laboratoires publics ou privés. 


\title{
Towards Haptic Images: a Survey on Touchscreen-Based Surface Haptics
}

\author{
Antoine Costes, Fabien Danieau, Ferran Argelaguet, Philippe Guillotel, Anatole Lécuyer
}

\begin{abstract}
The development of tactile screens opens new perspectives for co-located image and haptic rendering, leading to the concept of "haptic images". They emerge from the combination of image data, rendering hardware, and haptic perception. This enables one to perceive haptic feedback while manually exploring an image. This raises nevertheless two scientific challenges, which serve as thematic axes of the state of the art in this survey. Firstly, the choice of appropriate haptic data raises a number of issues about human perception, measurements, modeling and distribution. Secondly, the choice of appropriate rendering technology implies a difficult trade-off between expressiveness and usability.
\end{abstract}

Index Terms-survey, haptics, surface, touchscreen, image, texture.

\section{INTRODUCTION}

C ONCURRENTLY to the spreading of touchscreens, the interest in their haptic enhancement grew, and became a new research field called "surface haptics". Surface haptics refers to any system actuating a physical surface in order to produce haptic effects, preferably on the bare finger [1]. In the scope of this paper, we are mainly interested in the haptic rendering of images on touchscreens. We refer to this concept as "haptic images". Therefore, we will narrow this survey on surface haptics systems which provide a co-located visuohaptic feedback. The considered images can be 2D pictures as well as virtual objects in a 3D scene.

The premises of haptic images were laid with the concept of shape changing surfaces [2]. Ambitious attempts were made to mechanically actuate a surface in order to reproduce any shape in an interactive manner (see Section III-D). With the help of video projection, these displays provide co-localized shape and visual information. In these approaches, the resolution of the rendering is directly linked to the (high) density of actuators, resulting in a very expensive system.

The rise of tablet computers brought a new perspective to haptic images. They are highly integrated devices, combining touch tracking and visual display, in addition to self-contained battery power and operating system to run software. Therefore, it became very advantageous to use them as part of a haptic rendering setup. For instance, force-feedback arms or robotic systems were used to actuate directly a touchscreen, and provide it with force and motion abilities [3]. Most efforts in surface haptics have been concentrated on generating various types of vibrations that can alter the physics of the finger sliding on the screen, providing friction forces and even small relief sensations [4], [5]. Some alternative approaches made

A. Costes, F. Danieau and P. Guillotel are with InterDigital Rennes, France. F. Argelaguet and A. Lécuyer are with Inria Rennes, France. use of an intermediate proxy acting on the finger with minimal sight obstruction [6]. Also, crossmodal effects like pseudohaptic feedback can elicit haptic percepts without the need of a haptic actuator [7].

Figure 1 summarizes the scientific topics and challenges haptic images are related to. The conception of haptic images addresses image data, rendering hardware, as well as human haptic perception of surfaces. In this survey, we do not consider the issues related to image acquisition and rendering, which are broadly discussed in the literature. The concept of haptic images raises nevertheless two scientific challenges.

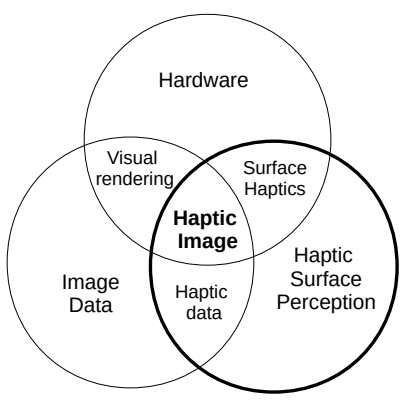

Fig. 1. Scientific fields and technical challenges for haptic images. The scope of the survey falls into the circle in bold.

Firstly, the general-case formalization of haptic data is not achieved yet. Surprising as it may seem, the very definition of the haptic properties of a material remains nowadays an open research question. Even in the engineering industry, there is no standard procedure (except for very specific contexts) to measure a set of properties needed to describe a material haptically.

Secondly, haptic rendering requires a set of technologies that can be complex to deploy. Covering a wide range of haptic sensations usually implies heavy technologies which are not likely to meet to consumer market. In order to get an interesting trade-off between technical complexity and expressiveness of the rendering, the development of haptic technologies requires to take into account perceptual factors and to focus on the most meaningful elements of haptic phenomena. As stated by Chang et al., "haptics are best employed when minimal actuation can have broad and great effect" [8].

\section{HAPTIC DATA: PERCEPTION, MEASUREMENTS, MODELING AND DISTRIBUTION}

The use of haptic data can be an issue to achieve haptic rendering. Haptic rendering is the production of sensory stimuli in response to user interactions in order to produce one or 
several haptic sensations (such as shape, compliance, friction, etc...) [9], [10]. While some systems are able to produce realistic sensations from simple mathematical heuristics, other make use of real-world measurements. Yet, haptic features are often tricky to characterize, and there is no standard way of measuring them, because there are no generalized definitions for them. Given the complexity of describing a subjective haptic experience, on which data should haptic simulation rely?

\section{A. The challenge of haptic data: formalizing haptic properties}

Every day, we touch and manipulate a large variety of materials, that we usually distinguish easily without the need of looking at them: our tactile sensitivity allows us to discriminate almost instantly a soft and sticky rubber from a cold and smooth metal or a harsh and dry textile. If identifying an object among others in a box can take only a few seconds [11], verbalizing the criteria on which we rely is much less spontaneous. In fact, there is no generic set of descriptors (verbal or not) to classify materials haptically. For instance, Yoshida had a quite wide approach with 20 adjective pairs [12]. On the other hand, when reducing the scope to a specific family, like banknotes, only two features can suffice for an accurate discrimination [13].

Because of a lack of appropriate vocabulary, tactile sensations and material properties are named with identical words (like "roughness" or "hardness"), although they are totally different things. This confusing polysemy gets even worse in the engineering field, where despite precise and formal definitions, a property like "roughness" can be described by a dozen of parameters and measured by several different methods [14]. As they relate to both physical properties and perceptual phenomena, haptic properties find themselves at the intersection of several fields of study like psychophysics, contact mechanics or surface metrology. Each of these perspectives may contribute, for a part, to the definition of a given haptic property, but they often have conflicting terminology.

Thus, the haptic description of an object often relies on subjective and context-dependent choices, which impedes the scientific effort of merging analysis and results. There is a need to clarify on which features one relies on when we appreciate or compare surfaces through touch. This would help to design haptic experiences more finely and achieve better user performance and experience, but also to refine the conception of haptic rendering devices. Haptic images directly raise this issue, with the concept of a generic visuo-haptic data format.

Once a measurable haptic data is defined, the design and fabrication of custom sensors is often needed. Adequate sensors for haptic measurements tend to be technically complex to conceive and expensive to produce. Their use is diversified and aims at different goals, namely robotic manipulation, haptic evaluation, material identification or realistic haptic simulations. Because each one of these application contexts implies a different use of haptic data, they require very different haptic acquisition approaches.

In this section, we first address the complexity of haptic perception of surfaces and the attempts to formalize it. Then, we review the different strategies used to produce haptic information from the capture of real objects features. We also address the question of haptic modeling and its reliance or not upon haptic measurements. Finally, we review recent attempts of making haptic data publicly available, which might be a decisive element for the development of haptic images.

\section{B. Semantic issues with haptic descriptors: the case of rough-} ness

Because they relate both to physical features and perceptual phenomena, haptic properties easily cross multiple perspectives and fields of study. Their restriction to quantitative characteristics leads inevitably to some terminology conflict. The term "roughness" is probably the most illustrative case of this semantic complexity.

Roughness has been extensively studied in the haptic literature (see [15] for a review), and yet its definition varies widely between authors. Even excluding perceptual aspects, roughness as a physical property is not a simple concept and has a debatable definition (according to the ISO 4287:1997, a dozen of different parameters can be used to assess it). Across this variety of conceptions, what can be safely said is that roughness is a geometrical feature of a surface, which relates to some form of spatial frequency.

Regardless of the chosen definition for "physical roughness", perceived roughness is supposed to vary in intensity according to the former. Perceived roughness has been firstly hypothesized to be a single quantity depending only on a few geometrical properties of the touched surface, but no convincing model was found despite considerable efforts [16], [17], [18], [19], [20], [21]. It was demonstrated later that the perceived roughness verbally expressed by different subjects would relate to different objective measurements [22]. This suggests that a plurality of perceptions is associated to the word "roughness". In other words, roughness entails several components and is hardly reducible to a single scalar value.

Indeed, a distinction is commonly made between fine and macro-roughness, which was confirmed by several studies [23], [24], [25] many decades after it was hypothesized by Katz under the famous name of the "duplex theory" [26]. This theory states that fine and coarse asperities are mediated by two distinct perceptual mechanisms, the first one relying on contact vibrations and the second one involving spatial distribution of pressure. It was notably found that contact vibrations are necessary to perceive asperities under $0.1 \mathrm{~mm}$ [25]. Costes et al. pointed out that the perceived roughness cannot describe entirely the surface geometry, in particular bumps or holes features [27]. Interestingly, a similar distinction can be found in metrology (ISO 4287:1997), where surface deviations are split on both sides of a sampling frequency: "roughness" refer to high frequency components, while low-frequency components are designated as "waviness".

In order to address such semantic issues, Okamoto et al. suggested to distinguish between different semantic layers: the perceived attributes of a material, which constitute the psychophysical layer, serve as an interface between the measurable physical properties of the material (the material layer), 
and higher cognitive layers (affective and preferential) [28]. The psychophysical layer relates to the perceptual representation of the touched object, and defines the scope for perceptual dimensionality, which we will address hereafter.

\section{Haptic perception of surfaces}

Haptic perception is part of the somatosensory system, which mediates sensations coming from the body tissues (like skin, muscles or viscera). Physical sensations originate from the nerve impulses sent by a variety of receptors, which are distributed in the body and are specific to each sensory system. These receptors are of different types, with various shape, constitution and distribution making them sensitive to specific stimulation. The receptors of the tactile sense fall into three categories: mechanoreceptors, thermoreceptors and nociceptors. Although temperature sensing can play a crucial role in tactile discrimination, mechanoreceptors are of higher interest in the present work, because from a technological perspective, surface haptics is much more prone to mechanical stimulation than thermal rendering. For more details regarding the characteristics of these receptors, we refer the reader to [29], [30], [31].

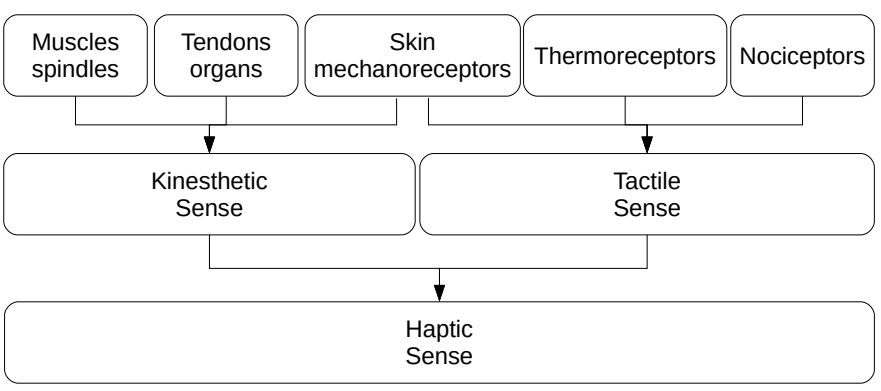

Fig. 2. Types of receptors for the kinesthetic and the tactile sensory systems.

\section{Perceptual dimensionality of haptic surfaces}

In daily life we touch many surfaces and in many cases, we can identify them in a second without the need of looking at them. On the other hand, it is sometimes hard to get a specific object out of the bottom of a bag without several trials and visual validation. What are the features on which our haptic representation of surfaces is based on?

This question has been addressed in a large body of studies (for a review, see [32]). While these studies are generally consistent about the two most discriminative features being roughness and stiffness (which definitions are variable though), the rest of their results are quite diversified, yet without strong contradictions. This can be explained by the fact that these studies vary a lot in terms of stimuli choice, psychophysical methods and mathematical methods [33]. Tiest and Kappers demonstrated that if many studies concluded in a 2- or 3dimensions model, they were valid only for a quite limited range of materials and that more descriptors were required to accurately depict the diversity of real-world materials [34]. Although most studies do not even mention it, temperature appears to be a very discriminative feature [35]. Stickiness was mentioned in more recent studies [36], [34]. In fact, there has been little common understanding for decades in haptic research about the dimensionality of touch perception.

The notion of "tactile primary colors" has been proposed by Kajimoto et al., emphasizing the complementarity of the mechanical stimuli which the four types of skin mechanoreceptors are sensitive to [37], [38]. As an analogy with the correspondence between color receptors in the eye and the color decomposition into primary colors, they suggested that a tactile sensation could be decomposed in four elementary stimuli, which would be perceived in a relatively independent manner. Pacini corpuscles react to the vibrations of a rough rubbing, Meissner corpuscles detect the indentation or stretch changes due to contact pressure, Merkel complexes are sensitive to the indentation due to a rough texture, while Ruffini endings are supposed to respond to shear deformations produced by adherence. However, this direct correspondence appears to be very simplistic: for instance it does not explain why hardness can be correctly estimated through tapping vibrations rather than squeezing pressure. It has been argued by that the central integration of tactile afferents in the primary somatosensory cortex does not reflect this submodalities decomposition, but rather higher-level neuronal representations of tactile features across different receptor types [39]. For instance, while the spatial pattern of SA-I activation accurately reflects the shape in contact with the finger, it has been shown that subjects were able to identify letters formed by vibrating patterns which activated RA-I and RA-II, but not SA-I afferents [40].

In order to address semantic issues, Okamoto et al. suggested to distinguish between different semantic layers: the perceived attributes of a material, which constitute the psychophysical layer, serve as an interface between the "objective" physical properties of the material (the material layer), and higher cognitive layers (affective and preferential) [28]. The psychophysical layer relates to the perceptual representation of the touched object, and defines the scope for perceptual dimensionality. They proposed to consider thermal dimension and three mechanical dimensions: compliance, roughness and friction, which is in accordance with other reviews [41], [42].

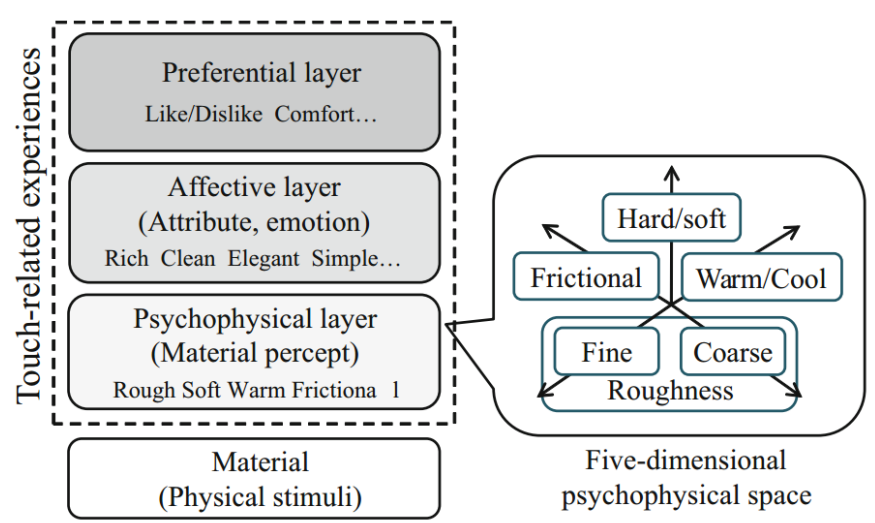

Fig. 3. Semantic layers of touch-related experiences, from [28].

Considered in a broad sense, compliance refers to how the surface deforms, roughness relates to its geometrical features, and friction concerns the easiness of the sliding 
against it. Yet, each one of these three reviews outlined the contradictory diversity of experimental findings, suggesting that these four dimensions remain general categories rather than clearly distinct features. The recent review of Costes et al. argued for distinguishing the modalities (vibratory, cutaneous, kinesthetic, thermal) from the percepts (compliance, friction, roughness and warmth) and considering ten elementary haptic features arising from their combination [43].

\section{E. From tactile sensing to haptic evaluation}

Tactile sensing, defined as the measurement of "given properties of an object through physical contact between the sensor and the object" [44], was developed in the first place for teleoperation systems, because reflecting contact forces is crucial for manipulation performances [45]. Semi or fully automated robotic manipulation also has a crucial need for tactile sensing, for instance to address the challenge of maintaining the grasp of an object with unknown weight and friction coefficient. Yet, these applications were in practice limited to real-time force and torque sensing, and did not ambition any storage of information for later use [44]. However, in the late nineties, the alternative use of tactile sensors to evaluate haptic properties was considered, pointing out many applications in other fields like medicine (especially for tumor detection), cosmetics (for product evaluation), or food industry (for delicate handling and inspection) [44]. For a comprehensive historical review, we refer the reader to Tiwana et al.'s review [46].

From that moment, the interest grew for devices able to haptically characterize an object, which is also much valuable for the field of realistic haptic rendering. The idea of "realitybased" haptic models spread widely during the 2000s, with the exemplary ACME system [47]. This robotic measurement facility could produce completely automated measurements and be controlled over the network. It included notably a laser range finder for shape acquisition, a stereo vision color system for reflectance acquisition, and a probe with a force and torque sensor for texture, friction and elasticity estimations.

However, a limitation of computer-controlled scanning systems is that they hardly reproduce the mechanical behavior of a human hand. Therefore, despite of their high reliability and reproducibility, they produce contact forces which differ from the one of natural human interaction. In this regard, several handheld probes were proposed instead [48], [49]. They measured contact forces and vibrations, and were usually combined with an optical position tracking system. Methods to map such surface measurements on a 3D mesh in an interactive manner were discussed in [50] and [51]. Battaglia et al. went even further and proposed thimble-shaped wearable sensors in order to measure multi-finger forces during grasping and manipulation [52].

\section{F. Bio-inspired sensors and material classification}

Artificial fingers constitute another trend for tactile sensor design [53], [54], [55], [56]. By mimicking the mechanical behavior of a human finger, they aim at reducing, as much as possible, the effect of artificial mechanical transductions which are typically induced with probes. Artificial fingers also get inspired by human mechanoreceptors [54], and most of them seek for multimodal sensing. An exception is the work of Edwards et al. who had an original approach using an inexpensive microphone only for textural discrimination [57]. However artificial fingers are not necessarily intended to capture data for realistic haptic rendering; they rather replace hands for material classification tasks [15]. In particular, the "BioTac" sensor distinguished itself by featuring a thermal sensing ability, in addition to vibration and deformation [56].

As machine learning techniques spread widely in almost all fields of science, the most recent trend in haptic acquisition is the treatment of unconstrained multimodal data. Gao et al. applied deep learning over both visual and haptic data to develop a prediction model for robots to anticipate contact with visible objects [58]. The "Proton Pack" is a handheld surface interaction recording system with an impressive number of sensors [59], [60], [61]. It is intended to produce an ambitious multimodal dataset for autonomous robots to properly interact with their environment. Another inspiring body of work in that domain is the one of Strese et al. who used audio signal analysis in conjunction with machine learning algorithms on both sound and acceleration data [62], [63]. One particularly interesting trait of their approach is that they put effort on establishing a ground truth about perceptual similarity from subjective psychophysical experiments, aiming at matching robotic analysis with the five human perceptual dimensions [64], [65].

\section{G. Explicit and implicit haptic modeling}

Haptic data are not necessarily at the core of haptic rendering, but haptic modeling is. Haptic rendering can be considered as a relationship between given exploratory or manipulatory inputs and given delivered outputs. In this regard, it expresses a model of the contact phenomena.

The most straightforward approaches for rendering consist in using explicit models: approximating the virtual object by an idealized, simplified mathematical model in order to run a physical simulation. The quality and realism of the resulting experience is thus dependent on the proper choice of the physical parameters of the model. The typical example is the use of the Hooke's law to simulate stiffness. Because of their simplicity, explicit models were popular in the beginnings of haptic rendering [66], [67], [68]. The parameters of the model can be eventually fitted through measurements done on real objects [69], [51], [70], [71], which is usually referred as "measurement-based modeling". Explicit models have two main limitations. Firstly, they require some preliminary knowledge on the physical phenomena that are to be simulated. Secondly, outside of simplistic cases, they often require considerable sophistication to provide a convincing result, and can thus be unfit for the real-time rendering of complex, realistic scenes with non-linear behaviors [72].

More recently, "data-driven" approaches were proposed as an alternative, in which the rendered forces are computed from an interpolation of sparse recordings of the real interaction [73], [74], [75], [76] . A strength of such implicit methods is to make use of precalculations on the recorded 
data to provide sophisticated outcomes in real-time. In a pioneer contribution, Hover et al. proposed two offline force field interpolation schemes in order to render one-dimensional visco-elasticity [74]. Their method could handle arbitrary nonlinear materials with visco-elastic behavior. This work was later extended to the rendering of viscous fluids [77], slipping phenomena [78], and inhomogeneous behaviors [79]. More recently, Costes et al. proposed a texture-based format to store ten haptic features [43]. It extends the concept of normal or bump maps used in computer graphics to add fine relief to $3 \mathrm{D}$ objects. Their textures can store friction, hardness, or temperature information that are mapped to 3D objects. Shin et Choi relied on photogrammetry to generate a high quality height map of set of textures [80]. Parametric models were used for friction and stiffness though (Dahl and Hunt-Crossley models respectively). Because of their promising possibilities in terms real-time realistic rendering, data-driven approaches received great attention in the field of medical training and robotic surgery [81], [71].

\section{H. Haptic databases}

A few authors worked on constituting haptic datasets for use by the research community. They are listed in Table II. Publicly accessible databases save the research community time because collecting data can be time intensive and expensive. This ambition is quite recent, as the very first attempt was the one of Culbertson et al. [82 1 . They provided one hundred haptic texture and friction models, the recorded data from which the models were made, images of the textures, and the code and methods necessary for rendering on a commercially available device. Each texture and friction model was based on a ten-second freehand recording of the force, speed, and highfrequency acceleration measured with an instrumented probe.

Shortly after, Strese et al. proposed a set of controlled and freehand acceleration recordings of 43 different textures [83]. Control recordings were done under two conditions, either with constant force and increasing scan velocity, or constant velocity and increasing normal force. Uncontrolled recordings consisted in a set of ten different twenty-second recordings following five lateral movements and five circular movements. The dataset was later extended to 69 textures, including measurements of sound, grasping force, and images [62]. The authors also provide on their websit ${ }^{2}$ two others databases of 108 surface materials and 184 buildings materials.

The Proton Pack ${ }^{3}$ project envisions the constitution of a comprehensive multimodal dataset [61]. Because of the quantity of data it is able to gather, this work opens a lot of exciting possibilities and challenges in data treatment and analysis. Another ambitious recent work foresees a "universal haptic library' 4 where psychophysical haptic features are matched with visual features, in order to automatically generate a haptic data-driven model from an unknown visual texture [73]. Such

${ }^{1}$ http://haptics.seas.upenn.edu/index.php/Research/

$$
\begin{aligned}
& \text { ThePennHapticTextureToolkit } \\
& { }^{2} \text { https://zeus./mt.ei.tum.de/downloads/texture/ } \\
& { }^{3} \text { https://alexburka.com/penn/proton.php } \\
& { }^{4} \text { http://haptics.khu.ac.kr/the-haptic-library/ }
\end{aligned}
$$

an approach tackles the difficulty of producing quality haptic content in large and complex scenes, which is one of the main issue of bringing haptics into immersive 3D environments.

Most of the databases contain records of the interaction of a tool with a surface. These data may not be appropriate for simulating interaction with the bare finger. Thus, Jioa et al. created the "HapTex" 5 that aggregate data of fingertips sliding over 120 pieces of fabric [84]. It includes friction force, friction coefficient, normal force, displacement and velocity of the fingertip. They showed how these data can be used with an electrostatic tactile display to simulate textures.

\section{TECHNOLOGICAL SOLUTIONS FOR TOUCHSCREEN-BASED SURFACE HAPTICS}

On a more materialistic perspective, one major limitation of haptic technologies is the technical complexity of mechanical actuators. If traditional force-feedback devices are effective for teleoperation and have been introduced in industrial and medical applications, they remain often complex, cumbersome and expensive. Adding more pieces of hardware to render more haptic properties will not help to spread this technology in the consumer market like touchscreens did.

\section{A. The challenge of surface haptics hardware: lightweight but expressive}

The challenge of simplicity applies to haptic technologies from their conception to their end-use, as its impact is considerable on both production costs and use case relevance. Usual vibrators embedded in cellphones and game controllers are a typical example of a successful simple technology, but their expressiveness is also remarkably limited. They illustrate on one hand the relevance of the haptic modality in many cases, and on the other hand the immense underuse of our haptic sensitivity.

Haptic researchers have proposed a number of innovative solutions in the recent years to provide haptic feedback and tactile sensations to touchscreens. Yet, most of these technologies provide only a limited range of tactile sensations, which depends largely on the generated stimulus. In order to address the full richness of haptic perception, several actuators could be combined to deliver specific stimuli of different kinds: forces, vibrations, shape and temperature. However the technical complexity of such a build-up can be huge.

Besides, it is noteworthy that an additional stimulus (for instance, shape in addition to forces) does not necessarily adds to the richness of the rendering. Before being transformed into a sensation, sensory cues are merged into a complex integration process [86], [87]. Hence, the quality and expressiveness of a haptic rendering is less due to the number of stimuli than to their congruence and complementarity; which is hard to evaluate directly. Some approaches can be used to estimate the optimal number of dimensions necessary to discriminate between samples, like multidimensional scaling [88]. They provide useful qualitative leads on the perceptual significance of each considered features, but do not provide definitive answers for the technical dilemmas of rendering technologies.

5 http://haptic.buaa.edu.cn/English_FabricDatabase.htm 


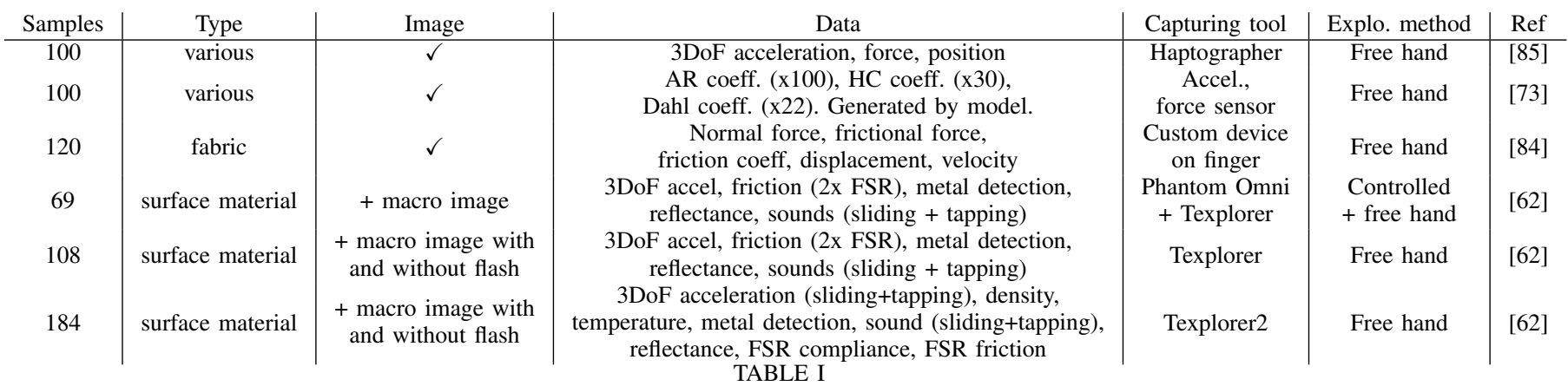

HAPTIC DATABASES ASSOCIATING IMAGES TO HAPTIC DATA.

In this section, we review solutions for the haptic enhancement of touchscreens according to the type of actuation they imply. The vast majority of them is based on mechanical stimulation, and neglects thermal stimuli. This can be explained by the technical difficulty to combine tactile screen interactions with temperature control. Although some examples exist [89], they are non-colocated and were therefore considered as out of scope for our review. These solutions are summarized in Table [II and organized along Costes et al.'s perceptual dimensions (see Section II-D).

\section{B. Vibrotactile actuators}

Because of their simplicity of integration, embedded vibrators are very common in nowadays mainstream tactile devices, and they tend to be intensively used for both gaming and GUI interactions enhancement. It is a fact that even a very simplistic haptic feedback can considerably increase the comfort and/or performance of tactile screen interactions (like the vibratory feedback when typing, for instance) [90]. However, the possibilities of usual embedded vibrators for haptic feedback remain limited: because they act on the whole screen as a single source, they produce a similar effect on different fingers touching the screen, and they cannot provide localized or moving stimuli. Furthermore, because they are generally simple eccentric rotors, they operate in a very narrow range of frequencies.

Many researchers have proposed creative ways to enrich touchscreens with an additional vibrator. The vibrator can be placed either on the nail [91], between several fingers and the screen [92], on the device [93], [94], [95] or both on the device and on haptic gloves [96]. In particular, Romano and Kuchenbecker used a high-quality one-dimensional vibration to display compelling texture details through an actuated stylus, according to normal contact force and lateral speed [97].

\section{Variable friction displays}

Vibrations can be used as a haptic signal mechanically transmitted to a finger; but they can also be a mean to modify the physical phenomena occurring on contact with a surface. In particular, ultrasonic frequencies are able to produce a thin film of air between the finger and the vibrating a surface, resulting in a diminished resistance to sliding. This friction reduction technique, first described by Watanabe and Fukui [98], was applied to touchscreens only in the 2010s [99], [100].
Another technique, called electrovibration, consists in amplifying electrostatic forces through high-voltage oscillations [101]. Although the concept was dating back to the 1950s [102], Linjama and Mäkinen were the first to use it on a transparent substrate, compatible with a tactile screen [103].

In both approaches, friction can be modulated to produce texture effects and even 3D pattern features [4], [5]. Smith and Gorlewicz combined both approaches on a single touchscreen to enlarge the range of simulated friction [104].

Planar force feedback rendering can be achieved with variable friction applied in an asymmetric way. For instance Chubb et al. applied in-plane oscillations to their device's friction pad while actively varying this pad's friction level to create directional shear forces [1].

\section{Shape changing screens}

Shape changing screens, as their name indicates, intend to reproduce the shape of an object consistently to its visual display. A pioneer work in that field is the FEELEX project [2], a deformable $24 \mathrm{~cm} \times 24 \mathrm{~cm} \times 3 \mathrm{~mm}$ plane actuated by an array of thirty six linear actuators. Each actuator is combined with a force sensor, providing interaction with the graphics which are projected on the surface from the top. Since then, a variety of other technological solutions have been proposed to achieve a similar concept. The main technical challenge for shape changing screens is to achieve acceptable performances for both resolution and actuation latency, with a limited bulkiness. Jansen et al. used electromagnets and magnetorheological fluids to achieve low-latency multitouch feedback, at the cost of a low resolution [105]. In contrast, Follmer et al.'s Tunable Clay is a malleable screen with a hydraulic-activated particle jamming controlling its global stiffness [106]. Leithinger et al. proposed a solution with simplified open-source hardware and no sensors in order to make it scalable and affordable [107]. In particular, the MIT Media Lab did an impressive work in designing a variety of pinbased shape displays and exploring their many applications, like UI dynamic affordance, physical rendering, 3D model manipulation, physical telepresence, music computing [108], [109], [110], [111]. Siu et al. extended the concept with a mobile tabletop display [112]. They designed a 288 actuated square pin array, mounted on a mobile platform. Relying on optical tracker, the device is able to follow one's hand watching a surface within a VR headset. 


\section{E. Moveable touchscreens}

As an alternative to the technical complexity of shape changing screens, it can be beneficial to actuate a regular touchscreen with a robotic system providing motion and/or force-feedback abilities. By doing so, one takes advantage of the highly integrated nature of touchscreens, namely a highresolution visual display with co-localized touch tracking. Yet, only a few solutions were proposed to haptically enhance a touchscreen with motion.

The "TouchMover" device is a touchscreen actuated and moved using force-feedback in the normal direction [113]. The second version includes vibrators that allows to render either fine shape details or local content information [114]. Another approach presented by Takanaka et al. consists in a touchscreen with planar force-feedback and large translation and rotation abilities, simulating contact, inertia, shape and stiffness [115]. In a way similar, Costes et al. proposed "KinesTouch", a tablet computer mounted on a 3DoF forcefeedback device [27]. In addition to shape, compliance, friction and fine roughness of a surface can be simulated.

Rotational movements can also be used to express the curvature of a virtual object on contact point, as proposed by Kim et al. [116]. Parallel platforms are an efficient way to provide this inclination feedback, as shown in the work of Maiero et al. [117] and Hausberger et al. [3].

\section{F. Actuated proxies}

Instead of actuating the touchscreen itself, several authors proposed to make use of an intermediate part. Most of these solutions can be classified along three categories: the one providing a proxy just under the finger, the one actuating a transparent overlay covering the screen, and the one acting on the back of a handheld device in a pseudo-colocated way. We will not address here the many works involving actuated probes (stylus, pen, stick, etc...), as they imply rather different types of interaction than touching the screen with the finger pad.

Portillo et al. proposed to use a pantograph above a touchscreen for multimodal handwriting and drawing assistance [118]. Yang et al. had an original approach with a 4-DoF string-based system actuating two digital rings and providing grasp force-feedback [119]. The system was adapted later on a handheld tablet, with a single ring, in order to provide $2.5 \mathrm{D}$ geometry and texture feedback [120], [6]. The "FingerFlux" uses electromagnetic actuation on a permanent magnet placed under the finger [121]. It achieves attraction, repulsion, vibration, and directional haptic feedback on and near the surface, perceivable at a distance of several centimeters. Finally, the "HapticLens" is a transparent "tangible" allowing to feel the stiffness of any region of an image [106]. The hydraulic jamming system controls the density of the chamber, going from liquid to solid consistency.

Some authors suggested to actuate a transparent film covering the whole screen. Wang et al. demonstrated a 2-DoF translational motion "Haptic Overlay Device", intended to enhance GUI interactions on automobile dashboards [122]. The inspiring work of Roudault et al. in particular, investigated the uses of a tactile gesture output [123]. Their two prototypes could guide the finger on the screen to reproduce a given gesture like a letter or a symbol, without the need of looking at it. Another approach is the one of Kajimoto who proposed a transparent electro-tactile display for active multi-touch shape feedback [124].

Finally, the "back-of-device approach" is a canny workaround for occlusion problems. Assuming the touchscreen is handheld, the position of a fingertip on its back side can be perceptually associated with the position of touch a input on the screen. Force feedback [125] or electro-tactile feedback [126] can be provided to a holding finger on its back side, in reaction to thumb touch inputs. In contrast, Kokobun et al. used the back side as a touch input to showcase visual pseudo-haptic effects without occlusion [127].

\section{G. Pseudo-haptic feedback}

"Pseudo-haptic feedback" refers to a non-haptic feedback inducing or modifying a haptic sensation in response to a force or motion input. Its very first use was to replace expensive haptic devices with passive ones [134]. The first contributions on the topic mostly relied on modifying the Control / Display (C/D) ratio of the mouse cursor in order to generate the feeling going through a bump or a hole. Additional works explored other haptic properties such as shape for stiffness, speed for friction or mass, trajectory for slope (for a review, see [134]).

In the following decade, alternative approaches were explored. Keller et al. induced "pseudo-weight" sensations in drag interactions by making object harder to displace [135]. Watanabe changed the C/D ratio of a scrolling background image instead of the cursor to induce friction sensations [128]. The "Elastic Image" proposed by Argelaguet et al. consists in deforming an image being clicked locally [129]. The progressive deformation (which approximately substitute pushing force by time) creates a quantitative softness sensation. With a similar visual effect, Punpongsanon et al. used videoprojection and finger-tracking to enhance the perceived softness of a real object [136]. Ban et al. used visual retargeting to provide illusory shape curvatures [137]. Only a few authors applied pseudo-haptic principles to touch interactions. Some of them chose a non-colocated approach, to avoid occlusion and decoupling issues [138] [127] [139].

Other authors leveraged co-localized touch interactions to display pseudo-haptic effects on touchscreens. Ridzuan et al. changed the visual aspect of the user's finger according to the applied pressure to simulate variable stiffness [130]. The approach of Watanabe was adapted to touchscreens in [131] and [132], inducing quantitative sliding sensations. Fleureau et al. adapted the Elastic Image technique to a tactile tablet, with an additional audio feedback to simulate roughness [7]. Costes et al. relied on a deformable cursor between the user's finger and a tablet computer to provide pseudo haptic sensations of hardness, friction, fine roughness, and macro roughness [133].

\section{DISCUSSION AND CONCLUSION}

The concept of haptic images constitute a pragmatic entry point for major scientific challenges of tomorrow's haptics 


\begin{tabular}{|c|c|c|c|c|c|c|}
\hline Approach & Technology & Ref. & Compliance & Friction & Shape & Roughness \\
\hline Vibrotactile & Vibrations & ActiveClick [90] & $\checkmark$ & & & \\
\hline Vibrotactile & Finger-mounted vibrations & [91] & & & $\checkmark$ & \\
\hline Vibrotactile & Finger-mounted vibrations & {$[92]$} & & & $\checkmark$ & \\
\hline Moveable screen & 1DoF Normal force-feedback & TouchMover [113] & & & $\checkmark$ & \\
\hline Moveable screen & 1DoF Normal force feedback + vibrations & TouchMover 2.0 [114] & $\checkmark$ & & $\checkmark$ & \\
\hline Moveable screen & 2DoF Lateral force feedback & {$[115]$} & $\checkmark$ & & $\checkmark$ & \\
\hline Moveable screen & 3DoF force feedback & ForceTab [117] & & & $\checkmark$ & \\
\hline Moveable screen & 3DoF force feedback & Kinestouch [27] & $\checkmark$ & $\checkmark$ & $\checkmark$ & $\checkmark$ \\
\hline Moveable screen & 3Dof Rotational force-feedback & [116] & & & $\checkmark$ & \\
\hline Moveable screen & 3Dof Rotational force-feedback & Surtics [3] & & & $\checkmark$ & \\
\hline Shape changing screen & Pin Array & Feelex [2] & & & $\checkmark$ & \\
\hline Shape changing screen & Pin Array & Reflief 107 & & & $\checkmark$ & \\
\hline Shape changing screen & Pin Array & inFORM [109] & & & $\checkmark$ & \\
\hline Shape changing screen & Pin Array & ShapeShift [112] & & & $\checkmark$ & \\
\hline Shape changing screen & Array electromagnet + MR fluid & MudPad [105] & $\checkmark$ & & $\checkmark$ & \\
\hline Shape changing screen & Hydrolic jamming system & Tunable clay [106] & $\checkmark$ & & $\checkmark$ & \\
\hline Actuated proxy & Cable proxy & FingViewer [119] & & & $\checkmark$ & \\
\hline Actuated proxy & Cable proxy & {$[120]$} & & & $\checkmark$ & \\
\hline Actuated proxy & Cable proxy & [6] & & & $\checkmark$ & $\checkmark$ \\
\hline Actuated proxy & Finger-mounted magnet & FingerFlux [121] & $\checkmark$ & & & \\
\hline Variable friction & 2DoF Lateral force feedback & longRangeOuija [123] & & $\checkmark$ & & \\
\hline Variable friction & Ultrasonic friction reduction & $\mathrm{TPad}[5]$ & & $\checkmark$ & & \\
\hline Variable friction & Ultrasonic friction reduction & LATPad 99] & & $\checkmark$ & & \\
\hline Variable friction & Ultrasonic friction reduction & Tpad Fire [100] & & $\checkmark$ & $\checkmark$ & \\
\hline Variable friction & Electrostatic friction amplification & TeslaTouch [101] & & $\checkmark$ & & \\
\hline Variable friction & Electrostatic friction amplification & TeslaTouch [4] & & & $\checkmark$ & \\
\hline Variable friction & Electrostatic friction amplification & E-sense [103] & & $\checkmark$ & & \\
\hline Pseudo-haptics & $\mathrm{C} / \mathrm{D}$ ratio & {$[128$} & & $\checkmark$ & & \\
\hline Pseudo-haptics & $\mathrm{C} / \mathrm{D}$ ratio & Elastic image [129] & $\checkmark$ & & & \\
\hline Pseudo-haptics & $\mathrm{C} / \mathrm{D}$ ratio & [130] & $\checkmark$ & & & \\
\hline Pseudo-haptics & $\mathrm{C} / \mathrm{D}$ ratio & [131] & & $\checkmark$ & & \\
\hline Pseudo-haptics & $\mathrm{C} / \mathrm{D}$ ratio & {$[132]$} & & $\checkmark$ & & \\
\hline Pseudo-haptics & $\mathrm{C} / \mathrm{D}$ ratio & Touchy [133] & $\checkmark$ & $\checkmark$ & & $\checkmark$ \\
\hline Pseudo-haptics & $\mathrm{C} / \mathrm{D}$ ratio + audio & 7 & $\checkmark$ & & & $\checkmark$ \\
\hline
\end{tabular}

SURFACE HAPTIC DEVICES ASSOCIATED to A TACTILE DisPlay. THEY ARE CLASSIFIED ALONG Costes ET AL. PERCEPTUAL DIMENSIONS [27]. ROUGHNESS ENCOMPASSES FINE AND MACRO ROUGHNESS, AND WAMRTH IS NOT CONSIDERED HERE.

techniques. Firstly, the formal definition of haptic properties is not mature enough to lead to a generic haptic format. Secondly, despite very creative research efforts to develop haptic hardware, the trade-off between expressiveness and real usability remains a difficult choice. Thus, this paper provided a literature review in perspective of these two major topics: data and hardware.

The perceptual mechanisms of haptic interactions with surfaces were presented and discussed. While skin mechanoreceptors show specific sensitivities to various tactile features, the choice of descriptors to quantify a haptic experience remains an open question. The experimental evaluation of haptic sensations is easily scrambled by semantic issues. If they can be classified along four general perceptual dimensions, namely compliance, roughness/geometry, friction and warmth, there is a need for a more precise typology. There is room for more sophisticated psychological research, notably to compare different modalities providing the same percept. For example, the relative importance of cutaneous and kinesthetic cues for stiffness perception has been studied [140], but vibrational cues have never been put in comparison. For each sensation, the psychophysical thresholds and relative importance of each types of cues could be investigated. This would provide informative knowledge on the optimal complementarity between haptic features and help the design of effective and parsimonious haptic rendering systems.

Beside the problem of matching human sensations, defining haptic data also involve measurements, and necessary relates to modeling choices. Haptic data can derive from mathematical models, measurements of real materials or a combination of both. The few existing haptic databases illustrate both the heterogeneity of approaches for haptic acquisition, and the recent trend of taking advantage of machine learning techniques to extract haptic data from measurements. The visual provided in the databases could also be used to investigate the interactions between the visual and the haptic modalities. For instance, if a haptic texture model is rendered together with the visual of another sample, to what extent does the perceived haptic properties change? The topic of haptic material acquisition is thus still an open research question, as both real-world measurements and synthesis models have strengths and limitations.

We provided an overview of previous solutions for touchscreen haptic enhancement, which span a variety of technological approaches: vibrotactile feedback, variable friction displays, shape changing screens, moveable touchscreens, or actuated proxies. Regardless of their technical complexity, most of them address a limited range of sensations. Crossmodal effects like pseudo-haptic feedback can also complement haptic feedback without the need of a haptic actuator. Eventually, the concept of haptic images will follow the evolution of image displays towards haptic holography [141]. 


\section{REFERENCES}

[1] E. C. Chubb, J. E. Colgate, and M. A. Peshkin, "Shiverpad: A glass haptic surface that produces shear force on a bare finger," IEEE Transactions on Haptics, vol. 3, no. 3, pp. 189-198, 2010.

[2] H. Iwata, H. Yano, F. Nakaizumi, and R. Kawamura, "Project feelex: adding haptic surface to graphics," in Proceedings of the 28th annual conference on Computer graphics and interactive techniques. ACM, 2001, pp. 469-476.

[3] T. Hausberger, M. Terzer, F. Enneking, Z. Jonas, and Y. Kim, "Surftics-kinesthetic and tactile feedback on a touchscreen device," in 2017 IEEE World Haptics Conference (WHC). IEEE, 2017, pp. $472-477$

[4] S.-C. Kim, A. Israr, and I. Poupyrev, "Tactile rendering of $3 \mathrm{~d}$ features on touch surfaces," in Proceedings of the 26th annual ACM symposium on User interface software and technology. ACM, 2013, pp. 531-538.

[5] L. Winfield, J. Glassmire, J. E. Colgate, and M. Peshkin, "T-pad: Tactile pattern display through variable friction reduction," in Second Joint EuroHaptics Conference and Symposium on Haptic Interfaces for Virtual Environment and Teleoperator Systems (WHC'07). IEEE, 2007, pp. 421-426.

[6] S. Saga and R. Raskar, "Simultaneous geometry and texture display based on lateral force for touchscreen," in 2013 World Haptics Conference (WHC). IEEE, 2013, pp. 437-442.

[7] J. Fleureau, Y. Lefevre, F. Danieau, P. Guillotel, and A. Costes, "Texture rendering on a tactile surface using extended elastic images and example-based audio cues," in International Conference on Human Haptic Sensing and Touch Enabled Computer Applications. Springer, 2016, pp. 350-359.

[8] A. Chang, J. Gouldstone, J. Zigelbaum, and H. Ishii, "Pragmatic haptics," in Proceedings of the 2nd international conference on Tangible and embedded interaction. ACM, 2008, pp. 251-254.

[9] K. Salisbury, D. Brock, T. Massie, N. Swarup, and C. Zilles, "Haptic rendering: Programming touch interaction with virtual objects," in Proceedings of the 1995 symposium on Interactive 3D graphics. ACM, 1995, pp. 123-130.

[10] K. Salisbury, F. Conti, and F. Barbagli, "Haptic rendering: introductory concepts," IEEE computer graphics and applications, vol. 24, no. 2, pp. 24-32, 2004

[11] R. L. Klatzky, S. J. Lederman, and V. A. Metzger, "Identifying objects by touch: An "expert system"," Perception \& psychophysics, vol. 37 , no. 4, pp. 299-302, 1985.

[12] M. YOSHIDA, "Dimensions of tactual impressions (1)," Japanese Psychological Research, vol. 10, no. 3, pp. 123-137, 1968.

[13] I. R. Summers, R. J. Irwin, and A. C. Brady, "Haptic discrimination of paper," in Human Haptic Perception: Basics and Applications. Springer, 2008, pp. 525-535.

[14] ISO 5627, "paper and board - determination of smoothness (bekk method)," 1995.

[15] J. A. Fishel and G. E. Loeb, "Bayesian exploration for intelligent identification of textures," Frontiers in neurorobotics, vol. 6, p. 4, 2012.

[16] S. J. Lederman, "Tactile roughness of grooved surfaces: The touching process and effects of macro-and microsurface structure," Perception \& Psychophysics, vol. 16, no. 2, pp. 385-395, 1974.

[17] _ - "The perception of surface roughness by active and passive touch," Bulletin of the Psychonomic Society, vol. 18, no. 5, pp. 253255,1981

[18] — "Tactual roughness perception: spatial and temporal determinants," Canadian Journal of Psychology/Revue canadienne de psychologie, vol. 37, no. 4, p. 498, 1983.

[19] S. J. Lederman and M. M. Taylor, "Fingertip force, surface geometry, and the perception of roughness by active touch," Perception \& Psychophysics, vol. 12, no. 5, pp. 401-408, 1972.

[20] T. Miyaoka, T. Mano, and M. Ohka, "Mechanisms of fine-surfacetexture discrimination in human tactile sensation," The journal of the acoustical society of America, vol. 105, no. 4, pp. 2485-2492, 1999.

[21] M. Taylor and S. J. Lederman, "Tactile roughness of grooved surfaces: A model and the effect of friction," Perception \& Psychophysics, vol. 17, no. 1, pp. 23-36, 1975.

[22] W. M. B. Tiest and A. M. Kappers, "Haptic and visual perception of roughness," Acta psychologica, vol. 124, no. 2, pp. 177-189, 2007.

[23] S. Bensmaïa and M. Hollins, "Pacinian representations of fine surface texture," Perception \& psychophysics, vol. 67, no. 5, pp. 842-854, 2005.

[24] M. Hollins, S. Bensmaïa, and R. Risner, "The duplex theory of tactile texture perception," in Proceedings of the 14th annual meeting of the international society for psychophysics. The International Society for Psychophysics Québec, Canada, 1998, pp. 115-121.
[25] M. Hollins and S. R. Risner, "Evidence for the duplex theory of tactile texture perception," Perception \& psychophysics, vol. 62, no. 4, pp. 695-705, 2000.

[26] D. Katz, "The world of touch (le krueger, trans.)," Mahwah. NJ: Rrlbaum.(Original work published 1925), 1989.

[27] A. Costes, F. Danieau, F. Argelaguet-Sanz, A. Lécuyer, and P. Guillotel, "Kinestouch: 3d force-feedback rendering for tactile surfaces," in International Conference on Virtual Reality and Augmented Reality. Springer, 2018, pp. 97-116.

[28] S. Okamoto, H. Nagano, and H.-N. Ho, "Psychophysical dimensions of material perception and methods to specify textural space," in Pervasive Haptics. Springer, 2016, pp. 3-20.

[29] R. S. Johansson and A. Vallbo, "Tactile sensibility in the human hand: relative and absolute densities of four types of mechanoreceptive units in glabrous skin." The Journal of physiology, vol. 286, no. 1, pp. 283 $300,1979$.

[30] K. B. Shimoga, "A survey of perceptual feedback issues in dexterous telemanipulation. ii. finger touch feedback," in Proceedings of IEEE Virtual Reality Annual International Symposium. IEEE, 1993, pp. 271-279.

[31] K. O. Johnson, "The roles and functions of cutaneous mechanoreceptors," Current opinion in neurobiology, vol. 11, no. 4, pp. 455-461, 2001.

[32] S. Okamoto, H. Nagano, and Y. Yamada, "Psychophysical dimensions of tactile perception of textures," IEEE Transactions on Haptics, vol. 6 , no. 1, pp. 81-93, 2012.

[33] J. Wu, N. Li, W. Liu, G. Song, and J. Zhang, "Experimental study on the perception characteristics of haptic texture by multidimensional scaling," IEEE transactions on haptics, vol. 8, no. 4, pp. 410-420, 2015.

[34] W. M. B. Tiest and A. M. Kappers, "Analysis of haptic perception of materials by multidimensional scaling and physical measurements of roughness and compressibility," Acta psychologica, vol. 121, no. 1, pp. $1-20,2006$.

[35] L. A. Jones and M. Berris, "Material discrimination and thermal perception," in 11th Symposium on Haptic Interfaces for Virtual Environment and Teleoperator Systems, 2003. HAPTICS 2003. Proceedings. IEEE, 2003, pp. 171-178

[36] K. J. Kuchenbecker, J. Romano, and W. McMahan, "Haptography: Capturing and recreating the rich feel of real surfaces," in Robotics Research. Springer, 2011, pp. 245-260.

[37] H. Kajimoto, N. Kawakami, S. Tachi, and M. Inami, "Smarttouch: Electric skin to touch the untouchable," IEEE computer graphics and applications, vol. 24, no. 1, pp. 36-43, 2004.

[38] V. Yem and H. Kajimoto, "Wearable tactile device using mechanical and electrical stimulation for fingertip interaction with virtual world," in 2017 IEEE Virtual Reality (VR). IEEE, 2017, pp. 99-104.

[39] H. P. Saal and S. J. Bensmaia, "Touch is a team effort: interplay of submodalities in cutaneous sensibility," Trends in neurosciences, vol. 37, no. 12 , pp. 689-697, 2014

[40] E. P. Gardner and C. I. Palmer, "Simulation of motion on the skin. i. receptive fields and temporal frequency coding by cutaneous mechanoreceptors of optacon pulses delivered to the hand," Journal of Neurophysiology, vol. 62, no. 6, pp. 1410-1436, 1989.

[41] A. M. Kappers and W. M. B. Tiest, "Haptic perception of material properties," Responsive Polymer Surfaces, pp. 225-250, 2017.

[42] W. M. B. Tiest, "Tactual perception of material properties," Vision research, vol. 50, no. 24, pp. 2775-2782, 2010.

[43] A. Costes, F. Danieau, F. Argelaguet, A. Lécuyer, and P. Guillotel, "Haptic material: A holistic approach for haptic texture mapping," in International Conference on Human Haptic Sensing and Touch Enabled Computer Applications. Springer, 2018, pp. 37-45.

[44] M. H. Lee, "Tactile sensing: new directions, new challenges," The International Journal of Robotics Research, vol. 19, no. 7, pp. 636-643, 2000.

[45] R. D. Howe, "Tactile sensing and control of robotic manipulation," Advanced Robotics, vol. 8, no. 3, pp. 245-261, 1993.

[46] M. I. Tiwana, S. J. Redmond, and N. H. Lovell, "A review of tactile sensing technologies with applications in biomedical engineering," Sensors and Actuators A: physical, vol. 179, pp. 17-31, 2012.

[47] D. K. Pai, J. Lang, J. Lloyd, and R. J. Woodham, "Acme, a telerobotic active measurement facility," in Experimental Robotics VI. Springer, 2000, pp. 391-400.

[48] D. K. Pai and P. Rizun, "The what: A wireless haptic texture sensor," in 11th Symposium on Haptic Interfaces for Virtual Environment and Teleoperator Systems, 2003. HAPTICS 2003. Proceedings. IEEE, 2003, pp. 3-9. 
[49] E. Ruffaldi, D. Morris, T. Edmunds, F. Barbagli, and D. K. Pai, "Standardized evaluation of haptic rendering systems," in 2006 14th Symposium on Haptic Interfaces for Virtual Environment and Teleoperator Systems. IEEE, 2005, pp. 225-232.

[50] S. Andrews and J. Lang, "Haptic texturing based on real-world samples," in 2007 IEEE International Workshop on Haptic, Audio and Visual Environments and Games. IEEE, 2007, pp. 142-147.

[51] J. Lang and S. Andrews, "Measurement-based modeling of contact forces and textures for haptic rendering," IEEE Transactions on Visualization and Computer Graphics, vol. 17, no. 3, pp. 380-391, 2010.

[52] E. Battaglia, G. Grioli, M. G. Catalano, M. Santello, and A. Bicchi, "Thimblesense: an individual-digit wearable tactile sensor for experimental grasp studies," in 2014 IEEE International Conference on Robotics and Automation (ICRA). IEEE, 2014, pp. 2728-2735.

[53] Y. Mukaibo, H. Shirado, M. Konyo, and T. Maeno, "Development of a texture sensor emulating the tissue structure and perceptual mechanism of human fingers," in Proceedings of the 2005 IEEE international conference on robotics and automation. IEEE, 2005, pp. 2565-2570.

[54] C. M. Oddo, M. Controzzi, L. Beccai, C. Cipriani, and M. C. Carrozza, "Roughness encoding for discrimination of surfaces in artificial activetouch," IEEE Transactions on Robotics, vol. 27, no. 3, pp. 522-533, 2011.

[55] Y. Tada, K. Hosoda, Y. Yamasaki, and M. Asada, "Sensing the texture of surfaces by anthropomorphic soft fingertips with multi-modal sensors," in Proceedings 2003 IEEE/RSJ International Conference on Intelligent Robots and Systems (IROS 2003)(Cat. No. 03CH37453), vol. 1. IEEE, 2003, pp. 31-35.

[56] R. Balasubramanian and V. J. Santos, The human hand as an inspiration for robot hand development. Springer, 2014, vol. 95.

[57] J. Edwards, J. Lawry, J. Rossiter, and C. Melhuish, "Extracting textural features from tactile sensors," Bioinspiration \& biomimetics, vol. 3, no. 3, p. 035002, 2008.

[58] Y. Gao, L. A. Hendricks, K. J. Kuchenbecker, and T. Darrell, "Deep learning for tactile understanding from visual and haptic data," in 2016 IEEE International Conference on Robotics and Automation (ICRA). IEEE, 2016, pp. 536-543.

[59] A. Burka, S. Hu, S. Helgeson, S. Krishnan, Y. Gao, L. A. Hendricks, T. Darrell, and K. J. Kuchenbecker, "Proton: A visuo-haptic data acquisition system for robotic learning of surface properties," in 2016 IEEE International Conference on Multisensor Fusion and Integration for Intelligent Systems (MFI). IEEE, 2016, pp. 58-65.

[60] A. Burka and K. J. Kuchenbecker, "How much haptic surface data is enough?" in 2017 AAAI Spring Symposium Series, 2017.

[61] A. Burka, A. Rajvanshi, S. Allen, and K. J. Kuchenbecker, "Proton 2: Increasing the sensitivity and portability of a visuo-haptic surface interaction recorder," in 2017 IEEE International Conference on Robotics and Automation (ICRA). IEEE, 2017, pp. 439-445.

[62] M. Strese, C. Schuwerk, A. Iepure, and E. Steinbach, "Multimodal feature-based surface material classification," IEEE transactions on haptics, vol. 10, no. 2, pp. 226-239, 2016.

[63] M. Strese, C. Schuwerk, and E. Steinbach, "Surface classification using acceleration signals recorded during human freehand movement," in 2015 IEEE World Haptics Conference (WHC). IEEE, 2015, pp. 214 219.

[64] M. Strese, Y. Boeck, and E. Steinbach, "Content-based surface material retrieval," in 2017 IEEE World Haptics Conference (WHC). IEEE, 2017, pp. 352-357.

[65] M. Strese, C. Schuwerk, and E. Steinbach, "On the retrieval of perceptually similar haptic surfaces," in 2015 Seventh International Workshop on Quality of Multimedia Experience (QoMEX). IEEE, 2015, pp. 1-6.

[66] C. Basdogan and M. A. Srinivasan, "Haptic rendering in virtual environments," in Handbook of virtual environments. CRC Press, 2002, pp. 157-174.

[67] M. D. R. Minsky, "Computational haptics: the sandpaper system for synthesizing texture for a force-feedback display," Ph.D. dissertation, Massachusetts Institute of Technology, 1995.

[68] C. B. Zilles and J. K. Salisbury, "A constraint-based god-object method for haptic display," in Proceedings 1995 IEEE/RSJ International Conference on Intelligent Robots and Systems. Human Robot Interaction and Cooperative Robots, vol. 3. IEEE, 1995, pp. 146-151.

[69] S. Greenish, V. Hayward, V. Chial, A. Okamura, and T. Steffen, "Measurement, analysis, and display of haptic signals during surgical cutting," Presence: Teleoperators \& Virtual Environments, vol. 11, no. 6 , pp. 626-651, 2002.
[70] A. M. Okamura, M. R. Cutkosky, and J. T. Dennerlein, "Reality-based models for vibration feedback in virtual environments," IEEE/ASME Transactions on Mechatronics, vol. 6, no. 3, pp. 245-252, 2001.

[71] C. Pacchierotti, D. Prattichizzo, and K. J. Kuchenbecker, "Cutaneous feedback of fingertip deformation and vibration for palpation in robotic surgery," IEEE Transactions on Biomedical Engineering, vol. 63, no. 2, pp. 278-287, 2015.

[72] I. Peterlik and L. Matyska, "An algorithm of state-space precomputation allowing non-linear haptic deformation modelling using finite element method," in Second Joint EuroHaptics Conference and Symposium on Haptic Interfaces for Virtual Environment and Teleoperator Systems (WHC'07). IEEE, 2007, pp. 231-236.

[73] A. Abdulali, R. Rakhmatov, T. Ogay, and S. Jeon, "Data-driven modeling and rendering of force responses from elastic tool deformation," Sensors, vol. 18, no. 1, p. 237, 2018.

[74] R. Hover, M. Harders, and G. Székely, "Data-driven haptic rendering of visco-elastic effects," in 2008 Symposium on Haptic Interfaces for Virtual Environment and Teleoperator Systems. IEEE, 2008, pp. 201208.

[75] C. Pacchierotti, D. Prattichizzo, and K. J. Kuchenbecker, "A datadriven approach to remote tactile interaction: from a biotac sensor to any fingertip cutaneous device," in International Conference on Human Haptic Sensing and Touch Enabled Computer Applications. Springer, 2014, pp. 418-424.

[76] S. Yim, S. Jeon, and S. Choi, "Data-driven haptic modeling and rendering of frictional sliding contact with soft objects for medical training," in 2014 11th International Conference on Ubiquitous Robots and Ambient Intelligence (URAI). IEEE, 2014, pp. 66-67.

[77] R. Hover, G. Kósa, G. Szekly, and M. Harders, "Data-driven haptic rendering-from viscous fluids to visco-elastic solids," IEEE Transactions on Haptics, vol. 2, no. 1, pp. 15-27, 2009.

[78] R. Höver and M. Harders, "Measuring and incorporating slip in datadriven haptic rendering," in 2010 IEEE Haptics Symposium. IEEE, 2010, pp. 175-182.

[79] A. Sianov and M. Harders, "Data-driven haptics: Addressing inhomogeneities and computational formulation," in 2013 World Haptics Conference (WHC). IEEE, 2013, pp. 301-306.

[80] S. Shin and S. Choi, "Geometry-based haptic texture modeling and rendering using photometric stereo," in 2018 IEEE Haptics Symposium (HAPTICS). IEEE, 2018, pp. 262-269.

[81] M. Sathia Narayanan, X. Zhou, S. Garimella, W. Waz, F. Mendel, and V. N. Krovi, "Data driven development of haptic models for needle biopsy phantoms," in ASME 2012 5th Annual Dynamic Systems and Control Conference joint with the JSME 2012 11th Motion and Vibration Conference. American Society of Mechanical Engineers Digital Collection, 2013, pp. 419-427.

[82] H. Culbertson, J. M. Romano, P. Castillo, M. Mintz, and K. J. Kuchenbecker, "Refined methods for creating realistic haptic virtual textures from tool-mediated contact acceleration data," in 2012 IEEE Haptics Symposium (HAPTICS). IEEE, 2012, pp. 385-391.

[83] M. Strese, J.-Y. Lee, C. Schuwerk, Q. Han, H.-G. Kim, and E. Steinbach, "A haptic texture database for tool-mediated texture recognition and classification," in 2014 IEEE International Symposium on Haptic, Audio and Visual Environments and Games (HAVE) Proceedings. IEEE, 2014, pp. 118-123.

[84] J. Jiao, Y. Zhang, D. Wang, X. Guo, and X. Sun, "Haptex: A database of fabric textures for surface tactile display," in 2019 IEEE World Haptics Conference (WHC). IEEE, 2019, pp. 331-336.

[85] H. Culbertson, J. J. L. Delgado, and K. J. Kuchenbecker, "One hundred data-driven haptic texture models and open-source methods for rendering on 3d objects," in 2014 IEEE Haptics Symposium (HAPTICS). IEEE, 2014, pp. 319-325.

[86] M. O. Ernst and M. S. Banks, "Humans integrate visual and haptic information in a statistically optimal fashion," Nature, vol. 415, no. 6870, p. 429, 2002.

[87] L. Dupin, V. Hayward, and M. Wexler, "Direct coupling of haptic signals between hands," Proceedings of the National Academy of Sciences, vol. 112, no. 2, pp. 619-624, 2015.

[88] N. Jaworska and A. Chupetlovska-Anastasova, "A review of multidimensional scaling (mds) and its utility in various psychological domains," Tutorials in quantitative methods for psychology, vol. 5, no. 1, pp. 1-10, 2009.

[89] H. Richter, D. Hausen, S. Osterwald, and A. Butz, "Reproducing materials of virtual elements on touchscreens using supplemental thermal feedback," in Proceedings of the 14th ACM international conference on Multimodal interaction. ACM, 2012, pp. 385-392. 
[90] M. Fukumoto and T. Sugimura, "Active click: tactile feedback for touch panels," in CHI'O1 Extended Abstracts on Human Factors in Computing Systems. ACM, 2001, pp. 121-122.

[91] H. Ando, E. Kusachi, and J. Watanabe, "Nail-mounted tactile display for boundary/texture augmentation," in Proceedings of the international conference on Advances in computer entertainment technology. ACM, 2007, pp. 292-293.

[92] D. Burch and D. Pawluk, "Using multiple contacts with textureenhanced graphics," in 2011 IEEE World Haptics Conference. IEEE, 2011, pp. 287-292.

[93] S. Brewster, S. Brewster, F. Chohan, and L. Brown, "Tactile feedback for mobile interactions," in Proceedings of the SIGCHI conference on Human factors in computing systems. ACM, 2007, pp. 159-162.

[94] N. Yannier, A. Israr, J. F. Lehman, and R. L. Klatzky, "Feelsleeve: Haptic feedback to enhance early reading," in Proceedings of the 33rd Annual ACM Conference on Human Factors in Computing Systems. ACM, 2015, pp. 1015-1024.

[95] S. Zhao, A. Israr, and R. Klatzky, "Intermanual apparent tactile motion on handheld tablets," in 2015 IEEE World Haptics Conference (WHC). IEEE, 2015, pp. 241-247.

[96] A. Israr, S. Zhao, K. McIntosh, J. Kang, Z. Schwemler, E. Brockmeyer, M. Baskinger, and M. Mahler, "Po2: augmented haptics for interactive gameplay," in ACM SIGGRAPH 2015 Emerging Technologies. ACM, 2015, p. 21.

[97] J. M. Romano and K. J. Kuchenbecker, "Creating realistic virtual textures from contact acceleration data," IEEE Transactions on haptics, vol. 5, no. 2, pp. 109-119, 2011.

[98] T. Watanabe and S. Fukui, "A method for controlling tactile sensation of surface roughness using ultrasonic vibration," in Proceedings of 1995 IEEE International Conference on Robotics and Automation, vol. 1. IEEE, 1995, pp. 1134-1139.

[99] V. Levesque, L. Oram, K. MacLean, A. Cockburn, N. D. Marchuk, D. Johnson, J. E. Colgate, and M. A. Peshkin, "Enhancing physicality in touch interaction with programmable friction," in Proceedings of the SIGCHI Conference on Human Factors in Computing Systems. ACM, 2011, pp. 2481-2490.

[100] J. Mullenbach, C. Shultz, A. M. Piper, M. Peshkin, and J. E. Colgate, "Tpad fire: Surface haptic tablet," Proc of HAID, 2013.

[101] O. Bau, I. Poupyrev, A. Israr, and C. Harrison, "Teslatouch: electrovibration for touch surfaces," in Proceedings of the 23nd annual ACM symposium on User interface software and technology. ACM, 2010, pp. 283-292.

[102] E. Mallinckrodt, A. Hughes, and W. Sleator Jr, "Perception by the skin of electrically induced vibrations." Science, 1953.

[103] J. Linjama and V. Mäkinen, "E-sense screen: Novel haptic display with capacitive electrosensory interface," in HAID 2009, 4th Workshop for Haptic and Audio Interaction Design, 2009.

[104] T. A. Smith and J. L. Gorlewicz, "Hue: A hybrid ultrasonic and electrostatic variable friction touchscreen," in 2017 IEEE World Haptics Conference (WHC). IEEE, 2017, pp. 635-640.

[105] Y. Jansen, T. Karrer, and J. Borchers, "Mudpad: localized tactile feedback on touch surfaces," in Adjunct proceedings of the 23nd annual ACM symposium on User interface software and technology. ACM, 2010, pp. 385-386.

[106] S. Follmer, D. Leithinger, A. Olwal, N. Cheng, and H. Ishii, "Jamming user interfaces: programmable particle stiffness and sensing for malleable and shape-changing devices," in Proceedings of the 25th annual ACM symposium on User interface software and technology. ACM, 2012 , pp. $519-528$.

[107] D. Leithinger and H. Ishii, "Relief: a scalable actuated shape display," in Proceedings of the fourth international conference on Tangible, embedded, and embodied interaction. ACM, 2010, pp. 221-222.

[108] A. Colter, P. Davivongsa, D. D. Haddad, H. Moore, B. Tice, and H. Ishii, "Soundforms: Manipulating sound through touch," in Proceedings of the 2016 CHI Conference Extended Abstracts on Human Factors in Computing Systems. ACM, 2016, pp. 2425-2430.

[109] S. Follmer, D. Leithinger, A. Olwal, A. Hogge, and H. Ishii, "inform: dynamic physical affordances and constraints through shape and object actuation." in Uist, vol. 13, 2013, pp. 417-426.

[110] D. Leithinger, S. Follmer, A. Olwal, and H. Ishii, "Physical telepresence: shape capture and display for embodied, computer-mediated remote collaboration," in Proceedings of the 27th annual ACM symposium on User interface software and technology. ACM, 2014, pp. 461-470.

[111] D. Leithinger, S. Follmer, A. Olwal, S. Luescher, A. Hogge, J. Lee, and H. Ishii, "Sublimate: state-changing virtual and physical rendering to augment interaction with shape displays," in Proceedings of the
SIGCHI Conference on Human Factors in Computing Systems. ACM, 2013, pp. 1441-1450.

[112] A. F. Siu, E. J. Gonzalez, S. Yuan, J. B. Ginsberg, and S. Follmer, "Shapeshift: 2d spatial manipulation and self-actuation of tabletop shape displays for tangible and haptic interaction," in Proceedings of the 2018 CHI Conference on Human Factors in Computing Systems. ACM, 2018, p. 291.

[113] M. Sinclair, M. Pahud, and H. Benko, "Touchmover: actuated 3d touchscreen with haptic feedback," in Proceedings of the 2013 ACM international conference on Interactive tabletops and surfaces. ACM, 2013, pp. 287-296.

[114] — "Touchmover 2.0-3d touchscreen with force feedback and haptic texture," in 2014 IEEE Haptics Symposium (HAPTICS). IEEE, 2014, pp. 1-6.

[115] S. Takanaka, H. Yano, and H. Iwata, "Multitouch haptic interface with movable touch screen," in SIGGRAPH Asia 2015 Haptic Media And Contents Design. ACM, 2015, p. 13.

[116] S.-C. Kim, B.-K. Han, J. Seo, and D.-S. Kwon, "Haptic interaction with virtual geometry on robotic touch surface," in SIGGRAPH Asia 2014 Emerging Technologies. ACM, 2014, p. 8.

[117] J. Maiero, E. Kruijff, A. Hinkenjann, and G. Ghinea, "Forcetab: Visuohaptic interaction with a force-sensitive actuated tablet," in 2017 IEEE International Conference on Multimedia and Expo (ICME). IEEE, 2017, pp. 169-174.

[118] O. Portillo, C. A. Avizzano, M. Raspolli, and M. Bergamasco, "Haptic desktop for assisted handwriting and drawing," in ROMAN 2005. IEEE International Workshop on Robot and Human Interactive Communication, 2005. IEEE, 2005, pp. 512-517.

[119] Y. Yang, Y. Zhang, Z. Hou, Z. Chen, and B. Lemaire-Semail, "Fingviewer: A new multi-touch force feedback touch screen," in 2011 IEEE International Conference on Consumer Electronics (ICCE). IEEE, 2011, pp. 837-838.

[120] S. Saga and K. Deguchi, "Lateral-force-based 2.5-dimensional tactile display for touch screen," in 2012 IEEE Haptics Symposium (HAPTICS). IEEE, 2012, pp. 15-22.

[121] M. Weiss, C. Wacharamanotham, S. Voelker, and J. Borchers, "Fingerflux: near-surface haptic feedback on tabletops," in Proceedings of the 24th annual ACM symposium on User interface software and technology. ACM, 2011, pp. 615-620.

[122] D. Wang, K. Tuer, M. Rossi, and J. Shu, "Haptic overlay device for flat panel touch displays," in 12th International Symposium on Haptic Interfaces for Virtual Environment and Teleoperator Systems, 2004. HAPTICS'04. Proceedings. IEEE, 2004, p. 290.

[123] A. Roudaut, A. Rau, C. Sterz, M. Plauth, P. Lopes, and P. Baudisch, "Gesture output: eyes-free output using a force feedback touch surface," in Proceedings of the SIGCHI Conference on Human Factors in Computing Systems. ACM, 2013, pp. 2547-2556.

[124] H. Kajimoto, "Skeletouch: transparent electro-tactile display for mobile surfaces," in SIGGRAPH 2012, 2012.

[125] J. Maiero, D. Eibich, E. Kruijff, A. Hinkenjann, W. Stuerzlinger, H. Benko, and G. Ghinea, "Back-of-device force feedback improves touchscreen interaction for mobile devices," IEEE Transactions on Haptics, vol. 12, pp. 483-496, 2019.

[126] S. Khurelbaatar, Y. Nakai, R. Okazaki, V. Yem, and H. Kajimoto, "Tactile presentation to the back of a smartphone with simultaneous screen operation," in Proceedings of the 2016 CHI Conference Extended Abstracts on Human Factors in Computing Systems, ser. CHI EA '16. New York, NY, USA: Association for Computing Machinery, 2016, p. 281-284. [Online]. Available: https://doi.org/10.1145/2851581.2889459

[127] A. Kokubun, Y. Ban, T. Narumi, T. Tanikawa, and M. Hirose, "Representing normal and shearing forces on the mobile device with visuohaptic interaction and a rear touch interface," in 2014 IEEE Haptics Symposium (HAPTICS). IEEE, 2014, pp. 415-420.

[128] J. Watanabe, "Pseudo-haptic sensation elicited by background visual motion," ITE Transactions on Media Technology and Applications, vol. 1, no. 2, pp. 199-202, 2013.

[129] F. Argelaguet, D. A. G. Jáuregui, M. Marchal, and A. Lécuyer, "Elastic images: Perceiving local elasticity of images through a novel pseudohaptic deformation effect," ACM Transactions on Applied Perception (TAP), vol. 10, no. 3, p. 17, 2013.

[130] M. B. Ridzuan, Y. Makino, and K. Takemura, "Direct touch haptic display using immersive illusion with interactive virtual finger," in International Conference on Human Haptic Sensing and Touch Enabled Computer Applications. Springer, 2012, pp. 432-444.

[131] Y. Ujitoko, Y. Ban, T. Narumi, T. Tanikawa, K. Hirota, and M. Hirose, "Yubi-toko: finger walking in snowy scene using pseudo-haptic tech- 
nique on touchpad," in SIGGRAPH Asia 2015 Emerging Technologies. ACM, 2015, p. 29.

[132] T. Narumi, Y. Ujitoko, Y. Ban, T. Tanikawa, K. Hirota, and M. Hirose, "Resistive swipe: Visuo-haptic interaction during swipe gestures to scroll background images on touch interfaces," in 2017 IEEE World Haptics Conference (WHC). IEEE, 2017, pp. 334-339.

[133] A. Costes, F. Argelaguet, F. Danieau, P. Guillotel, and A. Lécuyer, "Touchy: A visual approach for simulating haptic effects on touchscreens," Frontiers in ICT, vol. 6, p. 1, 2019.

[134] A. Lecuyer, S. Coquillart, A. Kheddar, P. Richard, and P. Coiffet, "Pseudo-haptic feedback: can isometric input devices simulate force feedback?" in Proceedings IEEE Virtual Reality 2000 (Cat. No. OOCB37048). IEEE, 2000, pp. 83-90.

[135] C. Keller, J. Bluteau, R. Blanch, and S. Coquillart, "Pseudoweight: Making tabletop interaction with virtual objects more tangible," in Proceedings of the 2012 ACM international conference on Interactive tabletops and surfaces. ACM, 2012, pp. 201-204.

[136] P. Punpongsanon, D. Iwai, and K. Sato, "Softar: Visually manipulating haptic softness perception in spatial augmented reality," IEEE transactions on visualization and computer graphics, vol. 21, no. 11, pp. 1279-1288, 2015.

[137] Y. Ban, T. Kajinami, T. Narumi, T. Tanikawa, and M. Hirose, "Modifying an identified curved surface shape using pseudo-haptic effect," in 2012 IEEE Haptics Symposium (HAPTICS). IEEE, 2012, pp. 211216.

[138] K. Nakakoji, Y. Yamamoto, N. Matsubara, and Y. Koike, "Tciex: an environment for designing and experiencing a variety of visuo-haptic sensory conflicts," in Proceedings of the 3rd IEEE VR2011 Workshop on PIVE 2011, number Pive, 2011, pp. 23-26.

[139] F. Argelaguet, T. Sato, T. Duval, Y. Kitamura, and A. Lécuyer, "Collaborative pseudo-haptics: Two-user stiffness discrimination based on visual feedback," in International Conference on Human Haptic Sensing and Touch Enabled Computer Applications. Springer, 2014, pp. $49-54$

[140] W. M. B. Tiest and A. M. Kappers, "Cues for haptic perception of compliance," IEEE Transactions on Haptics, vol. 2, no. 4, pp. 189199, 2009.

[141] R. Hirayama, D. M. Plasencia, N. Masuda, and S. Subramanian, "A volumetric display for visual, tactile and audio presentation using acoustic trapping," Nature, vol. 575, no. 7782, pp. 320-323, 2019.

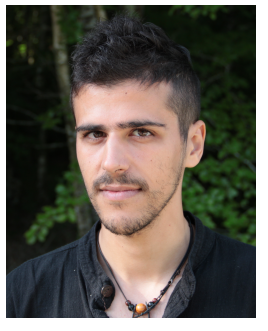

Antoine Costes is a Research Engineer at Inria Rennes, France. He obtained a $\mathrm{PhD}$ in Computer Science from INSA Rennes in 2018, within a collaboration between the Immersive Lab of InterDigital company (formerly Technicolor R\&I) and the Hybrid research team at Inria Rennes. His research topics include haptic enhancement of touchscreens, visuo-haptic interactions and pseudo-haptic rendering. He prototypes connected objects, interactive systems and haptic actuators for both scientific and artistic purposes.

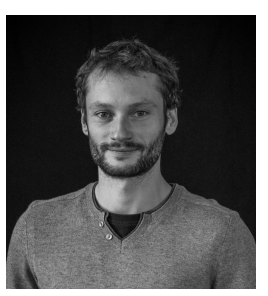

Fabien Danieau is a Researcher at InterDigital. His research focuses on the use of virtual reality to enhance the cinematographic experience. In particular he studies the impact of haptics and embodiment on the user experience. Before that he was researcher at Technicolor where he studied the use of new sensorial feedback to enhance the audiovisual experience. His main research interests include Haptics, Human Computer Interactions, Virtual Reality, Multimedia, User Experience.

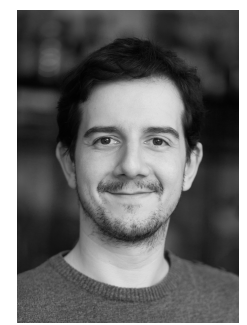

Ferran Argelaguet is a Research Scientist at the Hybrid research team, at Inria, the French National Institute for Research in Computer Science and Control, in Rennes, France. His research interests include 3D user interfaces, virtual reality and human perception. He received his $\mathrm{PhD}$ in Computer Science at the Polytechnic University of Catalonia in 2011. He is Program Chair of the IEEE Virtual Reality Conference track (2019-2020).

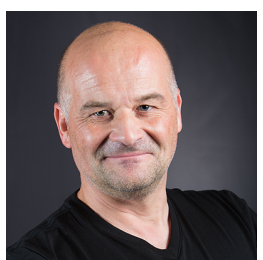

Philippe Guillotel Philippe Guillotel is Distinguished Scientist at InterDigital. He received the engineering degree in electrical engineering, telecommunications and computer science from the Ecole Nationale Superieure des Telecommunications (France) in 1988, the M.S. degree in electrical engineering and information theory from University of Rennes (France) in 1986, and the Ph.D. degree from University of Rennes (France) in 2012. His research interests include UX/user experiences (immersive experiences and user sensing), Human perception technologies (vision and haptics), video compression (2D and 3D) and $\mathrm{AI} /$ artificial intelligence (inc. robotics and machine/deep learning) for entertainment.

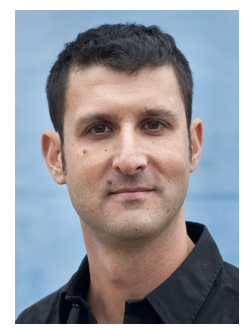

Anatole Lécuyer is Senior Researcher and head of Hybrid research team, at Inria, the French National Institute for Research in Computer Science and Control, in Rennes, France. His research interests include virtual reality, 3D interaction, haptic interfaces, and brain-computer interfaces. He is currently Associate Editor of "IEEE Transactions on Visualization and Computer Graphics", "Frontiers in Virtual Environments" and "Presence" journals, and formerly of "ACM Transactions on Applied Perception" and "IJHCS". He is author or co-author of more than 250 scientific publications. Anatole Lécuyer obtained the Inria-French Academy of Sciences "Young Researcher Prize" in 2013, and the IEEE VGTC "Virtual Reality Technical Achievement Award" in 2019. 